\title{
Is Cardiometabolic Index a Predictive Marker for Renal Cell Cancer Aggressiveness?
}

\author{
Murat Dursun ${ }^{1}$, Huseyin Besiroglu², Alper Otunctemur ${ }^{3}$, Emin Ozbek ${ }^{4}$ \\ ${ }^{1}$ Department of Urology, Malatya Training and Research Hospital, Malatya, Turkey; \\ ${ }^{2}$ Department of Urology, Catalca llyas Cokay State Hospital, Istanbul, Turkey; \\ ${ }^{3}$ Department of Urology, Okmeydani Training and Research Hospital, Istanbul, \\ Turkey; \\ ${ }^{4}$ Department of Urology, Istanbul Cerrahpasa Medical Faculty, Istanbul, Turkey \\ Received October 17, 2018; Accepted May 2, 2019.
}

Key words: Renal cell cancer - Cardiometabolic index - Metabolic syndrome Fuhrman grade

\begin{abstract}
Studies show that metabolic syndrome is a factor for developing renal cell cancer (RCC) and tumour aggressiveness. In our study, we evaluated the association between renal cell cancer and cardiometabolic index (CMI) which meets the main components of the metabolic syndrome. We retrospectively reviewed the records of 310 consecutive patients with RCC who underwent radical nephrectomy at our institution. We evaluated the tumour size, histologic subtype, Fuhrman nuclear grade. CMI was calculated as the product of waist circumference (WC) to waist-to-height ratio $(\mathrm{WH} t \mathrm{R})$ and triglyceride to high-density lipoprotein cholesterol ratio (TG/HDL-c). CMI were statistically higher in patients with tumour size $\geq 7 \mathrm{~cm}$ than those $<7 \mathrm{~cm}(\mathrm{p}<0.05)$. Mean $\mathrm{CMI}$ level was $2.34 \pm 0.84$ in patients with high tumour size; and $1.18 \pm 0.74$ in the other group. The patients with high tumour size had higher TG levels, higher WC and lower HDL-c levels. Similarly, CMI levels were statistically higher in patients with Fuhrman grade 3 and 4 than patients with Fuhrman grade 1 and $2(p<0.001)$. The patients with high Fuhrman grade had higher TG levels, higher WC and lower HDL-c levels. The simplicity of WC and height measurement and TG and HDL assessment make CMI an easily applicable index for the evaluation of cardiovascular dysfunction. The components of CMI may have effect on tumour carcinogenesis in similar pathways. In this context, CMI which meets the main components of the metabolic syndrome, can be a useful index for the evaluation and calculation of renal cell cancer aggressiveness.
\end{abstract}

Mailing Address: Murat Dursun, MD., Department of Urology, Malatya Training and Research Hospital, 44090, Turgut Özal Bulvarı, Yeşilyurt, Malatya, Turkey; Phone: +90 42249670 00; Fax: +90 42249670 00; e-mail: mrt_drsn@hotmail.com 


\section{Introduction}

Renal cell carcinoma (RCC) accounts for approximately $3 \%$ of all adult malignancies, representing the seventh most common cancer in men and the ninth most common cancer in women. Based on current guidelines, surgery remains the only curative treatment option in patients with localized RCC (Jones and Libermann, 2007; Edge and Compton, 2010; Ljungberg et al., 2015). It is a disease typically presenting in elderly patients with the mean age at diagnosis being around 60 years (Patard et al., 2004). Renal cell carcinoma originating from renal parenchyma is the majority of cases with kidney cancer. The predominant subtype of RCC is clear cell type that represents $80 \%$ of RCC and is derived from the tubular epithelium. Other types of RCC are papillary (15\%), chromophobe (5\%), and collecting duct (Shanks, 1999; Rodriguez et al., 2005).

Health risks are more associated with central obesity than total obesity. Based on this observation, many authors have proposed replacing BMI (body mass index) with waist circumference (WC) and/or waist-to-height ratio (WHtR) to determine the health risks of obesity and evaluating their use as metabolic syndrome screening tools (Nambiar et al., 2009; Ashwell et al., 2012). The ratio of triglycerides (TG) to HDL (high-density lipoprotein) cholesterol (TG/HDL-c) ratio has been proposed to be a good discriminator for cardiovascular risk (Gayziano et al., 1997; Jeppesen et al., 1997). TG/HDL-c ratio has been shown to reflect atherogenic small dense LDL (low-density lipoprotein) particles and to be associated with insulin resistance and metabolic syndrome (Cordero et al., 2008). In a study, a new index, named cardiometabolic index (CMI), calculated as the product of TG/HDL-c ratio and $\mathrm{WHtR}$, which are a good predictor of coronary artery disease and a central component of metabolic syndrome (Wakabayashi and Daimon, 2015). All of the components consisting of $\mathrm{CMI}$ are included in the criteria of metabolic syndrome (MetS). MetS is very common in developed countries, and its prevalence is likely to increase (Ford et al., 2002). Several mechanisms have been proposed to explain the relationship between MetS and malignant disease, including changes in neoplastic metabolism, DNA damage/repair malfunction, local inflammation and insulin-like growth factor 1 (Guo et al., 2013; Ozbek et al., 2014).

In our previous study, we reported that patients with metabolic syndrome were found to have statistically significant higher nuclear grade and tumour size (Ozbek et al., 2013). In overweight patients, visceral obesity has been shown to be a better marker than BMI in evaluating diseases such as cardiovascular diseases and metabolic syndrome. In this context, we thought that we could use the CMI for the assessment of RCC aggressiveness. The aim of this study was to evaluate the relationship between tumour size and grade with CMI levels in patients with renal cell carcinoma.

\section{Material and Methods}

We retrospectively reviewed the records of 310 consecutive patients with RCC who underwent radical nephrectomy at our institution between January 2007 and 
May 2015. We analysed the following clinicopathologic variables: age, gender, the presence of hypertension, diabetes, body mass index, waist circumference, height, tumour size, histologic subtype, Fuhrman nuclear grade, high-density lipoprotein and triglyceride levels. Plasma fasting glucose, high-density lipoprotein cholesterol levels and triglycerides were measured using enzymatic methods with an autoanalyzer. Pathologic staging was performed using the $7^{\text {th }}$ edition of the American Joint Committee on Cancer (AJCC). Histologic subtype was determined according to the 1997 World Health Organization Heidelberg classification and tumour nuclear grading was performed according to the Fuhrman nuclear grading system. BMI was calculated as weight in kilograms divided by the square of height in meters. CMI was calculated as the product of WHtR and TG/HDL-c ratio. Local ethics committee approval had been obtained before the commence of the study.

\section{Statistical analysis}

The baseline characteristics of the controls and the subjects were compared using a two sample $t$-test or Mann-Whitney $U$ test for the continuous variables. All statistical tests were two-tailed, and statistical significance was defined as $p<0.05$. All analyses were conducted using SPSS version 15.0 (SPSS Inc., Chicago, IL, USA).

\section{Results}

Among the 310 total patients analysed in our study, there were 176 males (56.8\%) and 134 females (43.2\%). We divided the patients in two groups (tumour size

\section{Table 1 - Characteristics of the patients and comparison to tumour size}

\begin{tabular}{lccr}
\hline & $\begin{array}{c}\text { Tumour size } \\
\geq 7 \mathrm{~cm}\end{array}$ & $\begin{array}{c}\text { Tumour size } \\
<7 \mathrm{~cm}\end{array}$ & P-value \\
\hline Age (mean-range) & $66.50 \pm 12.34$ & $62.80 \pm 8.94$ & 0.43 \\
BMI (mean \pm SD) & $28.36 \pm 2.87$ & $26.84 \pm 2.21$ & $<0.01$ \\
WC $(\mathrm{cm})($ mean $\pm \mathrm{SD})$ & $93.77 \pm 8.27$ & $91.03 \pm 8.51$ & $<0.01$ \\
Height (m) (mean \pm SD) & $1.68 \pm 0.42$ & $1.69 \pm 0.56$ & 0.88 \\
TG $(\mathrm{mg} / \mathrm{dl})($ mean $\pm \mathrm{SD})$ & $165.64 \pm 71.84$ & $129.58 \pm 72.21$ & $<0.01$ \\
$\mathrm{HDL}-\mathrm{c}(\mathrm{mg} / \mathrm{dl})($ mean $\pm \mathrm{SD})$ & $41.83 \pm 7.36$ & $47.54 \pm 4.28$ & $<0.01$ \\
$\mathrm{CMI}($ mean $\pm \mathrm{SD})$ & $2.34 \pm 0.84$ & $1.18 \pm 0.74$ & $<0.01$ \\
\hline
\end{tabular}

BMI - body mass index; WC - waist circumference; TG - triglycerides; HDL-c - high-density lipoprotein cholesterol; $\mathrm{CMI}$ - cardiometabolic index; SD - standard deviation

Table 2 - Correlation cardiometabolic index levels and Fuhrman grade

\begin{tabular}{lccc}
\hline & Fuhrman grade 1-2 & Fuhrman grade 3-4 & P-value \\
\hline CMI (mean \pm SD) & $1.04 \pm 0.56$ & $2.63 \pm 0.92$ & $<0.01$ \\
\hline
\end{tabular}

$\mathrm{CMI}$ - cardiometabolic index; SD - standard deviation 
$\geq 7 \mathrm{~cm}$ and $<7 \mathrm{~cm}$ ). BMI were statistically higher in patients with tumour size $\geq 7 \mathrm{~cm}$ than $<7 \mathrm{~cm}(p<0.001)$. Comparison of the patients with tumour size, the patients with high tumour size had higher TG levels, higher WC and lower HDL-C levels. The difference was statistically significant for all values. Also, we compared the CMI levels between two groups. Mean CMI level was $2.34 \pm 0.84$ in group 1 and $1.18 \pm 0.74$ in group 2 , respectively. CMI levels were statistically higher patients with high tumour size $(p<0.001)$. Characteristics of all people are shown in Table 1.

Also, we compared the CMI levels with Fuhrman grade. We divided the patients into two groups for Fuhrman grade. Fuhrman grade 1 and 2 was group 1, Fuhrman grade 3 and 4 was group 2 . BMI were statistically higher in patients with higher Fuhrman grade $(p<0.001)$. Comparison of the patients with Fuhrman grade, the patients with high Fuhrman grade had higher TG levels, higher WC and lower HDL-C levels. The difference was statistically significant for all values. Also, we compared the CMI levels between two groups. Mean CMI level was $2.63 \pm 0.92$ in group 2 and $1.04 \pm 0.56$ in group 1 , respectively. CMI levels were statistically higher in men and women with high Fuhrman grade $(p<0.001)$ (Table 2$)$. On the other hand, binary logistic regression models showed that $\mathrm{CMI}$ is an independently risk factor for renal cell cancer aggressiveness with higher Fuhrman grade and higher tumour size.

\section{Discussion}

In our study we investigated the correlation between tumour size and grade with cardiometabolic index in renal cell carcinoma. CMI, consisting of indices of adiposity and blood lipids: WHtR and TG/HDL-c ratio, respectively. The components of these indices are included in routine health check-up examinations and are easy to measure. All of the components consisting of $\mathrm{CMI}$ are included in the criteria of MetS. MetS is very common in developed countries, and its prevalence is likely to increase (Ford et al., 2002). There is increasing evidence to indicate that MetS might play an important role in the etiology and progression of certain types of cancers. Several mechanisms have been proposed to explain the relationship between MetS and malignant disease, including changes in neoplastic metabolism, DNA damage/repair malfunction, local inflammation and insulin-like growth factor 1 (Guo et al., 2013; Ozbek et al., 2014).

In a recent epidemiologic study from Northern Europe, Häggström et al. (2013) found that high levels of several metabolic factors, both separately and combined, were associated with an increased risk of RCC. Those metabolic factors were high levels of BMI, glucose, TG among men and BMI among women. Obesity causes the changes in lipid regulation and results in insulin resistance that may foster cancer development (Guo et al., 2013). Epidemiological studies performed to date have consistently shown an increased relative risk of RCC and the risk of RCC mortality with increases in BMI (Ibrahim, 2010; Zhang et al., 2018). Especially, increased visceral fat was found to be associated with clear-cell RCC. This study showed that, 
visceral fat area could constitute a primary explanation for the link between obesity and clear cell RCC (Bergström et al., 2001).

One of the components of CMI; waist-to-height ratio ( $\mathrm{WHtR}$ ) is an alternative tool instead of BMI or WC for identifying people at higher metabolic risk. It has been reported that $\mathrm{WHtR}$ is superior to $\mathrm{BMI}$ in detecting cardiovascular risk factors in adults (Lee et al., 2008). There is a concern that the cardiometabolic risk may differ between people who have the same WC yet differ in height. It was reported that shorter people have higher cardiometabolic risk than taller people with the same WC (Hsieh and Yoshinaga, 1999). However, there were no significant differences in odds ratios of any health risks between short people and tall people from the fourth quartile of waist circumference.

Height should be taken into account when defining obesity in children and adolescents, and the $\mathrm{WHtR}$ is an alternative parameter that considers height. WHtR seems to be useful in discriminating those at higher metabolic risk, even when it is used in combination with WC, likely due to its consideration of height. In a previous study visceral obesity as defined by VAT\% was found to be an independent prognostic factor for higher Fuhrman grade in patients with cT1a RCC. In this cohort of 186 patients with cT1a RCC and found that VAT\% was an independent prognostic factor for high grade disease after adjusting for clinical characteristics and anatomical features of the tumour (Zhu et al., 2013). Like this study, we found that CMl levels were statistically higher in men and women with high Fuhrman grade.

On the other hand, there are many studies validating the contribution of dyslipidemia to carcinogenesis of various cancers. An elevated risk of esophageal cancer and colon cancer was observed in people with high levels of TG (Inoue et al., 2009; Wulaningsih et al., 2012), and hypercholesterolemia has been suggested as a risk factor for rectal cancer (Wulaningsih et al., 2012). Little research has been conducted which directly focuses on the association between RCC and lipid disorders. In a prospective cohort study, Van Hemelrijck et al. (2012) reported that TG was the only lipid component for which a statistically significant association was observed with RCC. Also, statins (inhibitors of 3-hydroxy-3-methylglutaryl coenzyme A reductase) that are used for the treatment of lipid disorders, especially hypercholesterolemia, appear to protect against the development of RCC. Horiguchi et al. (2008) reported that fluvastatin had a notable inhibitory effect in vitro on tumour growth, invasion, angiogenesis, and metastasis of RCC cells, which provided indirect evidence of the relationship between dyslipidemia and RCC.

The mechanisms that underlie the role of $\mathrm{CMI}$ or MetS on RCC carcinogenesis are complicated, involving insulin resistance (IR), inflammation, angiogenesis, cellstroma interaction, and many other important aspects. Insulin is one of the most important anabolic hormones that is able to stimulate cell proliferation. Harmful local and systemic reactions, including intracellular lipid accumulation in adipocytes, mitochondrial and endoplasmic reticulum stress, and IR, can be induced by obesity, 
accompanied by changes of circulating factors such as adipokines, free fatty acids and a variety of inflammatory mediators. The insulin-like growth factor (IGF) family and the changes of its components resulting from IR play a crucial role in tumour formation and progression (Hernandez et al., 2015). Also, as a typical alteration in obesity, both hyperplasia and hypertrophy of adipocytes may lead to tissue hypoxia, and subsequently the induction of a series of pro-inflammatory cytokines. It has been shown that both IL-6 and IL-10 are strongly expressed in RCC cells and stroma. Further, IL-10 levels were higher in more advanced (PT3) tumours, suggesting that IL-6 and IL-10 may be useful markers associated with the development and progression of RCC (Cardillo and Ippoliti, 2007).

In conclusion, the components of CMI may have effect on tumour carcinogenesis in similar pathways. Especially, visceral obesity causes the changes in lipid regulation and results in insulin resistance that may foster cancer development. There are many studies validating the contribution of dyslipidemia to carcinogenesis of various cancers. In our study patients with high CMI levels were found to have statistically significant higher nuclear grade and tumour size. So, the simplicity of WC and height measurement and TG and HDL-c assessment make CMI an easily applicable index for the evaluation of cardiovascular dysfunction. CMI can be a useful index for the evaluation and calculation of renal cell cancer aggressiveness. Further studies with more patients are needed to confirm our study.

\section{References}

Ashwell, M., Gunn, P., Gibson, S. (2012) Waist-to-height ratio is a better screening tool than waist circumference and BMI for adult cardiometabolic risk factors: systematic review and meta-analysis. Obes. Rev. 13(3), 275-286.

Bergström, A., Hsieh, C. C., Lindblad, P., Lu, C. M., Cook, N. R., Wolk, A. (2001) Obesity and renal cell cancer - a quantitative review. Br. J. Cancer 85, 984-990.

Cardillo, M. R., Ippoliti, F. (2007) Interleukin-6, interleukin-10 and heat shock protein-90 expression in renal epithelial neoplasias and surrounding normal-appearing renal parenchyma. Int. J. Immunopathol. Pharmacol. 20, 37-46.

Cordero, A., Laclaustra, M., León, M., Casasnovas, J. A., Grima, A., Luengo, E., Ordoñez, B., Bergua, C., Bes, M., Pascual, I., Alegría, E.; MESYAS Registry Investigators (2008) Comparison of serum lipid values in subjects with and without the metabolic syndrome. Am. J. Cardiol. 102, 424-428.

Edge, S. B., Compton, C. C. (2010) The American Joint Committee on Cancer: the $7^{\text {th }}$ edition of the AJCC cancer staging manual and the future of TNM. Ann. Surg. Oncol. 17(6), 1471-1474.

Ford, E. S., Giles, W. H., Dietz, W. H. (2002) Prevalence of the metabolic syndrome among US adults: findings from the third national health and nutrition examination survey. JAMA 287, 356-359.

Gayziano, J. M., Hennekens, C. H., O’Donnell, C. J., Breslow, J. L., Buring, J. E. (1997) Fasting triglycerides, high-density lipoprotein, and risk of myocardial infarction. Circulation 96, 2520-2525.

Guo, Y. Z., Pan, L., Du, C. J., Ren, D. Q., Xie, X. M. (2013) Association between C-reactive protein and risk of cancer: a meta-analysis of prospective cohort studies. Asian Pac. J. Cancer Prev. 14, 243-248.

Häggström, C., Rapp, K., Stocks, T., Manjer, J. Bjørge, T., Ulmer, H., Engeland, A., Almqvist, M., Concin, H., Selmer, R., Ljungberg, B., Tretli, S., Nagel, G., Hallmans, G., Jonsson, H., Stattin, P. (2013) Metabolic factors associated with risk of renal cell carcinoma. PLoS One 8(2), e57475. 
Hernandez, A. V., Pasupuleti, V., Benites-Zapata, V. A., Thota, P., Deshpande, A., Perez-Lopez, F. R. (2015) Insulin resistance and endometrial cancer risk: A systematic review and meta-analysis. Eur. J. Cancer 51(18), 2747-2758.

Horiguchi, A., Ito, K., Sumitomo, M., Kimura, F., Asano, T., Hayakawa, M. (2008) Decreased serum adiponectin levels in patients with metastatic renal cell carcinoma. Jpn. J. Clin. Oncol. 38, 106-111.

Hsieh, S. D., Yoshinaga, H. (1999) Do people with similar waist circumference share similar health risks irrespective of height? Tohoku J. Exp. Med. 188, 55-60.

Ibrahim, M. M. (2010) Subcutaneous and visceral adipose tissue: structural and functional differences. Obes. Rev. 11, 11-18.

Inoue, M., Noda, M., Kurahashi, N., Iwasaki, M., Sasazuki, S., Iso, H., Tsugane, S. (2009) Impact of metabolic factors on subsequent cancer risk: results from a large-scale population-based cohort study in Japan. Eur. J. Cancer Prev. 18, 240-247.

Jeppesen, J., Hein, H. O., Suadicani, P., Gyntelberg, F. (1997) Relation of high TG-low HDL cholesterol and LDL cholesterol to the incidence of ischemic heart disease. An 8-year follow-up in the Copenhagen Male Study. Arterioscler. Thromb. Vasc. Biol. 17, 1114-1120.

Jones, J., Libermann, T. A. (2007) Genomics of renal cell cancer: the biology behind and the therapy ahead. Clin. Cancer Res. 13(2 Pt 2), 685s-692s.

Lee, C. M., Huxley, R. R., Wildman, R. P., Woodward, M. (2008) Indices of abdominal obesity are better discriminators of cardiovascular risk factors than BMI: a meta-analysis. J. Clin. Epidemiol. 61, 646-653.

Ljungberg, B., Bensalah, K., Canfield, S., Dabestani, S., Hofmann, F., Hora, M., Kuczyk, M. A., Lam, T., Marconi, L., Merseburger, A. S., Mulders, P., Powles, T., Staehler, M., Volpe, A., Bex, A. (2015) EAU guidelines on renal cell carcinoma: 2014 update. Eur. Urol. 67(5), 913-924.

Nambiar, S., Truby, H., Abbott, R. A., Davies, P. S. W. (2009) Validating the waist-height ratio and developing centiles for use amongst children and adolescents. Acta Paediatr. 98(1), 148-152.

Ozbek, E., Otunctemur, A., Sahin, S., Dursun, M., Besiroglu, H., Koklu, I., Polat, E. C., Erkoc, M., Danis, E., Bozkurt, M. (2013) Renal cell carcinoma is more aggressive in Turkish patients with the metabolic syndrome. Asian Pac. J. Cancer Prev. 14(12), 7351-7354.

Ozbek, E., Otunctemur, A., Dursun, M., Koklu, I., Sahin, S., Besiroglu, H., Erkoc, M., Danis, E., Bozkurt, M. (2014) Association between the metabolic syndrome and high tumor grade and stage of primary urothelial cell carcinoma of the bladder. Asian Pac. J. Cancer Prev. 15, 1447-1451.

Patard, J. J., Tazi, H., Bensalah, K., Rodriguez, A., Vincendeau, S., Rioux-Leclercq, N., Guillé, F., Lobel, B. (2004) The changing evolution of renal tumours: a single center experience over a two-decade period. Eur. Urol. 45, 490-493.

Rodriguez, C., Patel, A. V., Mondul, A. M., Jacobs, E. J., Thun, M. J., Calle, E. E. (2005) Diabetes and risk of prostate cancer in a prospective cohort of US men. Am. J. Epidemiol. 161, 147-152.

Shanks, J. H. (1999) Pathology of renal cell carcinoma: recent developments. Clin. Oncol. (R. Coll. Radiol.) 11, 263-268.

Van Hemelrijck, M., Garmo, H., Hammar, N., Jungner, I., Walldius, G., Lambe, M., Holmberg, L. (2012) The interplay between lipid profiles, glucose, BMI and risk of kidney cancer in the Swedish AMORIS study. Int. J. Cancer 130, 2118-2128.

Wakabayashi, I., Daimon, T. (2015) The "cardiometabolic index" as a new marker determined by adiposity and blood lipids for discrimination of diabetes mellitus. Clin. Chim. Acta 438, 274-278.

Wulaningsih, W., Garmo, H., Holmberg, L., Hammar, N., Jungner, I., Walldius, G., Van Hemelrijck, M. (2012) Serum lipids and the risk of gastrointestinal malignancies in the Swedish AMORIS study. J. Cancer Epidemiol. 2012, 792034. 
Zhang, J., Chen, Q., Li, Z. M., Xu, X. D., Song, A. F., Wang, L. S. (2018) Association of body mass index with mortality and postoperative survival in renal cell cancer patients, a meta-analysis. Oncotarget $\mathbf{9 ( 1 7 ) ,}$ 13959-13970.

Zhu, Y., Wang, H. K., Zhang, H. L., Yao, X. D., Zhang, S. L., Dai, B., Shen, Y. J., Liu, X. H., Zhou, L. P., Ye, D. W. (2013) Visceral obesity and risk of high grade disease in clinical T1a renal cell carcinoma. J. Urol. 189(2), 447-453. 